\title{
Bacterial orthologues of Ro60 trigger disease
}

Orthologues of Ro60, an evolutionarily conserved RNA-binding protein, are found in commensal bacteria that colonize the human skin, gut and oral mucosa. According to a new study published in Science Translational Medicine, these orthologues can elicit the production of autoantibodies that cross-react with human Ro60, thereby triggering autoimmune diseases such as systemic lupus erythematosus (SLE).

The idea of a potential pathogenic cause for SLE is not new. Antibodies directed against Ro60 are known to precede the onset of disease and previous studies in the lab of co-corresponding author Sandra Wolin have shown that many bacteria contain a protein that is strikingly similar to human Ro60. "When we discovered these bacterial Ro60 proteins, we suspected that they might be important for triggering autoimmunity," she explains.

"Infection by bacteria as inducers of Ro60 antibodies, as proposed by the field, would be transient," says co-corresponding author Martin Kriegel. "Commensal bacteria, on the other hand, that persist in the human body, are ideal candidates for not only initiating but also perpetuating anti-Ro60 autoimmunity."

By screening databases specifically for Ro60 orthologues in commensal bacterial species, the research team identified candidate species at many anatomical locations, including the skin, gut and airways. The presence of one

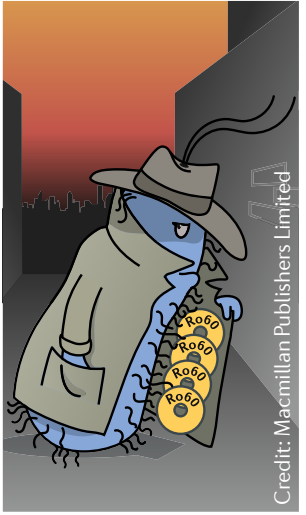

or more of four of these species was then confirmed at these sites in patients with SLE.

Kriegel and colleagues demonstrated functional cross-reactivity between human $\mathrm{T}$ cell clones isolated from patients with SLE and Ro60 orthologues from Propionibacterium propionicum and from Bacteroides thetaiotaomicron. Next, the researchers monocolonized germfree mice with $B$. thetaiotaomicron and induced lupus-like disease. Over time, they found lupus nephritislike disease in these mice, as well as increased titres of anti-human Ro60 antibodies.

Wolin and Kriegel believe that such commensal-induced autoimmunity could also be involved in diseases such as Sjögren syndrome, in which patients also produce anti-Ro60 antibodies.

Joanna Collison

\section{the onset of} disease

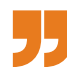

ORIGINAL ARTICLE Greiling, T. M. et al. Commensal orthologs of the human autoantigen Ro60 as triggers of autoimmunity in lupus. Sci. Transl Med. 10, eaan2306 (2018)

\section{OSTEOARTHRITIS}

\section{Fyn-ding a target for OA therapy}

The Src family kinase (SFK) Fyn phosphorylates $\beta$-catenin, causing nuclear localization, an activation signal in chondrocytes that results in degradation of cartilage and osteoarthritis (OA), according to a study published in Annals of the Rheumatic Diseases.

High levels of both $\beta$-catenin and WNT signalling are already associated with degradation of cartilage and $\mathrm{OA}$, but therapies that target specific arms of these pathways to enable disease modification are not available.

In the new study, the researchers analysed cartilage from 12-month-old mice, as an ageing model of $\mathrm{OA}$, with 2-month-old mice as controls. From a proteomics screen they identified Fyn as a major differentially expressed protein, being almost 12.5 times more abundant in cartilage of the older mice, with most Fyn accumulating inside chondrocytes. They also noted an increase in $\beta$-catenin activation signals.

Expression and nuclear localization patterns of Fyn match higher OA Research Society International (OARSI) scores and an increase in cartilage degeneration in these mice. By contrast, 12-month-old Fyn KO mice had less cartilage degradation than wild-type mice. The researchers also surgically induced destabilization of the medial meniscus (DMM) of wild-type and Fyn $\mathrm{KO}$ mice, with similar results to those in the ageing model.

This study confirms that, like other cell types, Fyn also activates $\beta$-catenin in chondrocytes and thereby identifies Fyn as a potential therapeutic target for OA. The researchers demonstrate this potential by treating (DMM) mice with AZD0530 (a SFK inhibitor) and PP1 (a Fyn and ATP-binding inhibitor).

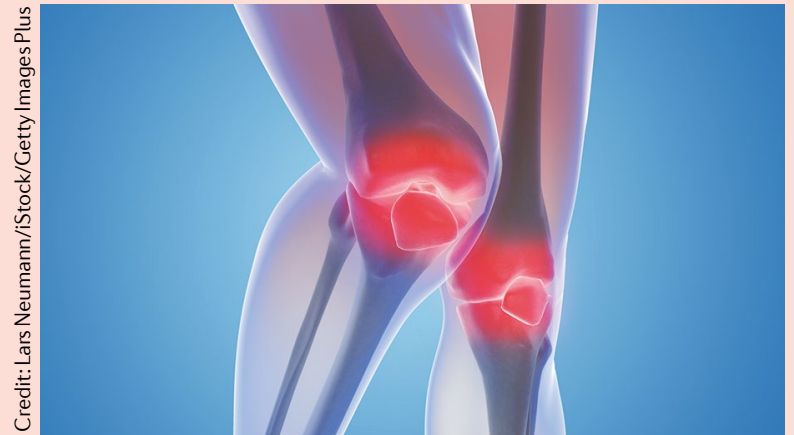

Whether Fyn is a suitable target for human intervention is not yet clear, particularly given the lack of Fyn specificity of the inhibitors. However, the study includes preliminary human data, including an analysis of cartilage from a small cohort of seven patients with OA compared with cartilage from four patients who underwent surgery for knee trauma. Similar to the mouse model data, accumulation of Fyn in cartilage was positively associated with OA.

Nicholas J. Bernard

intervention in not yet clear
ORIGINAL ARTICLE Li, K. et al. Tyrosine kinase Fyn promotes osteoarthritis by activating the $\beta$-catenin pathway. Ann. Rheum. Dis. https://doi. org/10.1136/annrheumdis-2017-212658 (2018) 